\title{
Transatlantica
}

Revue d'études américaines. American Studies Journal

1 | 2017

Morphing Bodies: Strategies of Embodiment in

Contemporary US Cultural Practices

\section{New York-Paris No.3 de Stuart Davis : dialogues transatlantiques}

\section{Kamila Benayada}

\section{(2) OpenEdition}

\section{Journals}

Édition électronique

URL : https://journals.openedition.org/transatlantica/8447

DOI : $10.4000 /$ transatlantica. 8447

ISSN : 1765-2766

Éditeur

Association française d'Etudes Américaines (AFEA)

Référence électronique

Kamila Benayada, "New York-Paris No.3 de Stuart Davis : dialogues transatlantiques », Transatlantica

[En ligne], 1 | 2017, mis en ligne le 27 novembre 2018, consulté le 20 mai 2021. URL : http://

journals.openedition.org/transatlantica/8447 ; DOI : https://doi.org/10.4000/transatlantica.8447

Ce document a été généré automatiquement le 20 mai 2021.

\section{cc) (†)}

Transatlantica - Revue d'études américaines est mise à disposition selon les termes de la licence

Creative Commons Attribution - Pas d'Utilisation Commerciale - Pas de Modification 4.0 International. 


\section{New York-Paris No.3 de Stuart Davis : dialogues transatlantiques}

Kamila Benayada

Stuart Davis (1892-1964), New York-Paris No 3, 1931

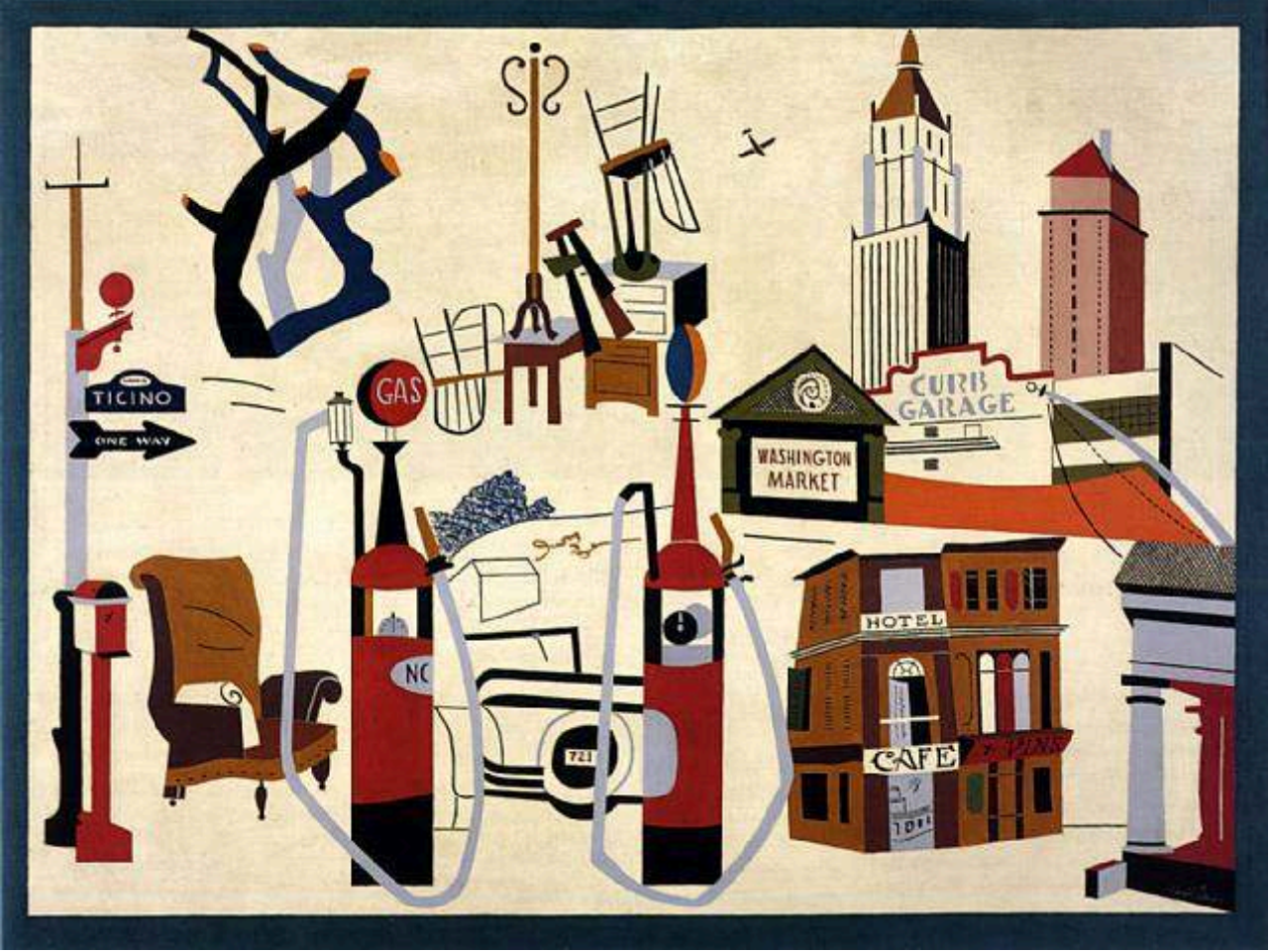

Huile sur toile, $99,5 \times 132,1 \mathrm{~cm}$

COLLECTION PARTICULIÈRE, DR. 


\section{Introduction}

En 1992, année du centième anniversaire de la naissance du peintre américain Stuart Davis, le Metropolitan Museum of Art organisait une exposition rétrospective de son œuvre intitulée «Stuart Davis, American Painter ». Plus récemment, en 2003, le musée d'art américain de Giverny montait « Paris, Capitale de l'Amérique ». La couverture de son catalogue arborait une reproduction de la toile New York-Paris No 1, et son titre évoquait celui choisi en 1987 par Whitney Museum of American Art : «Stuart Davis : an American in Paris ». Les titres de ces expositions suggèrent que Davis est perçu non seulement comme Américain, mais aussi comme représentatif de la communauté artistique américaine expatriée à Paris ${ }^{1}$. Cette double identification, cette double réception de l'œuvre de Davis sont symptomatiques de la dualité de son esthétique, à la fois transnationale et américaine, et cette dualité est justement mise en évidence dans son travail de 1931 dans la série des New York-Paris.

2 La carrière de Stuart Davis, qui débute avec le nouveau siècle, coïncide également avec des périodes de nationalisme exacerbé aux États-Unis ; il apparait déjà dans le domaine pictural au début du $\mathrm{xx}^{\mathrm{e}}$ siècle avec notamment les artistes dits de la poubelle, ou "Ashcan artists ", puis en 1913, par exemple, dans le cadre de l'exposition de l'armurerie (Armory Show). Dans les années 1920, la critique d'art met en avant une esthétique américaine, accompagnant les mouvements nativistes ${ }^{2}$. Au cours de cette décennie, le critique d'art Thomas Craven défend l'idée d'un art purement américain, libéré des influences européennes, représenté par les peintres régionalistes ou de la scène américaine, et avant tout par le peintre Thomas Hart Benton, surtout pendant la Grande Dépression. Puis le nationalisme dans l'art et la critique évoluent à l'encontre de ces régionalistes. L'œuvre de Davis part à la fois de ces nationalismes, qui prennent la forme d'un discours anti-européen, puis s'y oppose, dès sa découverte lors de l'Armory Show du modernisme venu d'outre-Atlantique.

3 Davis décide alors de devenir "a modern artist " (Davis, 1945, 13), à partir de cette confrontation avec l'École de Paris, et de produire un art qui serait une expression universelle et objective (" a universal objective statement », Davis, Stuart Davis Papers [SDP], juillet 1943). Son passage à Paris, de juin 1928 à août 1929, a été perçu comme une parenthèse dans son développement, avec un retour à la représentation figurative après une série de toiles où il montre qu'il maîtrise l'abstraction (les Egg Beaters de 1927-28 LIEN : http://collection.whitney.org/object/476). Cependant, alors que Davis peint dans un contexte où l'opposition Europe/Amérique est constante, ces peintures sont une zone de contact et de communication entre ces deux pôles. Son affiliation affirmée à Paris, qui fait de lui un " exilé » dans son propre pays (ce sont ses propres mots), le place dans une position critique anti-hégémonique, où le refus du nationalisme participe d'une stratégie de libération et de problématisation de son travail. L'incertitude et la réévaluation sont au centre de sa démarche de peintre et le placent dans ce que Gerard Delanty a appelé une position cosmopolite critique (Delanty, 2006). Le sociologue voit le cosmopolitisme comme une forme de contestation culturelle où la logique de la «translation » joue un rôle fondamental.

Dans le cas de Davis, il me semble que c'est précisément vers cela que sa peinture tend, en comprenant le mot 'translation' comme signifiant aussi «traduction», Davis étant un «traducteur » du cubisme. La traduction est donc ici une communication en même temps qu'un transfert, un passage, un glissement, un dé-placement, et donc un 
mouvement. À partir de cette série des trois New York-Paris ${ }^{3}$, Davis reprend et retravaille, de façon critique et dans la continuité, des toiles de jeunesse en les plaçant dans un contexte double, français et américain. Il s'approprie un langage pictural autre, il l'emploie contre le langage dominant, mais aussi il le transforme de façon à créer un espace à la fois dans la scène artistique, et à ses marges, en dehors, d'où un positionnement critique et contestataire devient possible.

5 La série New York- Paris est peinte en 1931, et, la même année, la galerie d'Edith Halpert organise une exposition Davis ayant le même titre. C'est donc que celui-ci importe à l'artiste. Deux ans après le krach de Wall Street, Herbert Hoover, l'homme de l'Amérique profonde, est élu à la présidence du pays, après une campagne où son adversaire démocrate, l'Irlandais catholique de New York Alfred E. Smith avait été accusé de vouloir installer le Pape à la Maison Blanche. Davis semble toutefois oublier le contexte politique, économique et social, et se pencher plutôt sur le souvenir de son année parisienne : il était parti pour la France en juin 1928 et était rentré à New York en août 1929. Le titre de ces toiles, ainsi que de l'exposition solo, suggère une certaine nostalgie. Pourtant, cette série est aussi, on le verra, le lieu d'une recherche esthétique qui est aussi une réflexion idéologique contestataire, où Davis se positionne contre le nationalisme, que la crise sociale qui suit le krach a exacerbé, et en défenseur d'une esthétique moderniste qui est alors condamnée car importée d'Europe : l'abstraction. Il détourne la scène américaine au profit d'une recherche esthétique fondée sur une interprétation personnelle du cubisme.

6 Peindre les trois New York-Paris en 1931 n'est donc pas anodin. Ce n'est pas non plus un refus de la part de l'artiste d'intégrer son environnement social et idéologique au profit d'une simple recherche esthétique. Mais dans ces toiles, et notamment la troisième, il y a aussi, et peut-être d'abord, une réflexion sur le cheminement professionnel de l'artiste. Elles ont parfois été perçues comme un retour en arrière pour Davis, après les abstractions de 1928, essentiellement à cause de l'aspect figuratif des œuvres; elles sont pourtant une étape essentielle dans la trajectoire du peintre.

\section{New York-Paris No 3 : un auto-portrait}

7 Si l'on examine cette toile, on y trouve, en suivant la flèche qui indique la direction à prendre, l'expression "one way», ou sens unique. Viennent ensuite des vignettes représentant un réverbère, deux arbres enchevêtrés, des meubles, des gratte-ciels à l'arrière du marché Washington et d'un garage, une boîte aux lettres et un fauteuil, deux pompes à essence avec une voiture au milieu et un paysage ébauché en arrièreplan, un hôtel qui fait aussi café, et une bâtisse à colonnes. De tous ces éléments, un seul est "parisien », l'hôtel-café-débit de boisson ("Vins»). Dans le contexte de la Prohibition, Davis, qui fréquentait les speakeasies ou bars clandestins apparus après l'adoption de la loi Volstead, suggère que Paris est un lieu de liberté, qui contraste avec la politique répressive des États-Unis des années 1920 ou années «folles». Si les femmes américaines bénéficient enfin du droit de vote, l'époque voit se développer un anticommunisme et un anti-anarchisme institutionnels, qui se traduisent par les rafles dans les milieux de gauche, et par une législation réduisant l'immigration de ressortissants des pays d'Europe du sud, du centre, et de l'est.

New York-Paris No 3 est donc avant tout une toile new-yorkaise, peinte par un artiste new-yorkais : les gratte-ciels, le marché (situé sur la 3e avenue), le restaurant Ticino 
(italien) de Greenwich Village, que Davis fréquentait, et la bâtisse à colonne qui a été identifiée comme étant la bourse de New York, lieu du krach et point de départ de la Grande Dépression en 1929.

9 Le tableau est aussi un résumé des thèmes et des formes chers à Davis, et que l'on trouve dans des toiles précédentes, et à venir. Les différents éléments sont une série d'auto-citations : les deux arbres, le garage, le lampadaire, les meubles, l'avion, les pompes à essence, le mât ont tous migré d'autres toiles antérieures vers cette œuvre, qui devient une sorte de résumé d'éléments à la fois narratifs et structurels que Davis retient dans son travail. Ils viennent, pour l'essentiel, de toiles représentant la ville de Gloucester, Massachusetts, que Davis identifie comme des toiles de la "Scène Américaine" (titre de son exposition chez E. Halpert en 1932). Davis entend bien récupérer l'appellation choisie par les régionalistes pour leur peinture " américaine ».

Ces éléments sont prétextes à un travail structurel de composition. Par exemple, Davis dispose des objets verticaux que sont ici le réverbère, les troncs d'arbre, les pompes à essence, le porte-manteau ou les gratte-ciel, qui lui permettent de structurer l'espace de la toile pour y organiser les autres éléments de la composition. Les deux troncs d'arbre bleu et noir encadrent le tronc bleu-ciel; les chaises retournées sont placées de part et d'autre du porte-manteau. Les gratte-ciel ne sont pas que l'arrière-plan, en contre-point, du marché et du garage; ils sont deux éléments verticaux qui contrebalancent le plan horizontal formé par le garage et le marché. Les pompes à essence encadrent l'arrière de la voiture et le paysage en ébauche. Les deux plans qui forment la façade de l'hôtel entourent l'entrée de l'hôtel où l'on distingue autre chose (un navire? une autre bâtisse ?). Et le poteau électrique qui se fond dans le réverbère, qui se fond lui-même dans la boîte aux lettres et son ombre, est une limite verticale à la composition, qui se rajoute au cadre peint autour de la toile, et le mur de droite du décor que forment le garage et le marché. Davis, donc, prend des éléments qui semblent disparates et contribuent moins à une narration claire qu'à dessiner des éléments structurels de la composition.

11 L'aspect fragmentaire qui en résulte est renforcé par l'emploi de lettres ou de mots, alors qu'on pourrait d'abord croire qu'ils participent à l'aspect narratif de la toile, en introduisant le verbe dans l'image: Ticino, à gauche, renvoie au restaurant de Greenwich Village. Le garage, le marché ou l'hôtel-café sont explicitement identifiés par Davis. Et ces indications renvoient à d'autres garages ou cafés dans des toiles précédentes. Mais d'autres mots apparaissent, de manière un peu inattendue : le garage s'appellerait «CURB » (soit le bord d'un trottoir, mais aussi un frein); et sous le nom du restaurant, Davis indique le sens à suivre, qui est un sens unique («ONE WAY »), donc un frein supplémentaire à la liberté de circuler, ainsi que de créer. C'est aussi un sens presque imposé de lecture de la toile, qui constitue une sorte d'entrave à la liberté de voir, et fait écho à d'autres entraves à la liberté aux États-Unis, imposées par l'Administration Wilson pendant la Grande Guerre dans le but de renforcer le sentiment anti-allemand : interdiction d'enseigner la langue ou interdiction de jouer de la musique allemande, par exemple.

Davis, amateur de J.S. Bach, autant que de jazz, joue du contrepoint dans sa construction formelle qui ressemble à une partition à plusieurs voix, avec renvois, échos et reprises, tant dans les formes que dans les couleurs. On trouve par exemple des indications similaires dans deux toiles de 1922, Landscape, Gloucester (où l'on retrouve le «garage» de Garage de 1917), et Landscape (Curve - Go Slow), mais 
«curve» ("virage») anticipe «curb», et "go slow» (« ralentir») annonce " one way» («sens unique »). Cette toile de 1922 est l'une des œuvres où Davis se lance dans l'expérimentation cubiste, s'inspirant des collages et du travail sur la fragmentation, la simultanéité et la surface plane de la toile. Elles se présentent comme des paysages (de Gloucester, Massachusetts), mais ces tableaux font déjà le pont entre Paris et les ÉtatsUnis.

De la même manière, les arbres de la première vignette sont les deux arbres de Early American Landscape (Whitney, 1925) que Davis rapproche et superpose. Dans cette première toile, les arbres servent de cadre à ce paysage de la première Amérique. Dans la première vignette de New York-Paris No.3, ils encadrent un autre tronc d'arbre qui, par le jeu des couleurs, apparaît en arrière-plan. Dans ce tableau, comme dans les deux Landscape de 1922 ou cet Early American Landscape, Davis fragmente le propos, joue sur la profondeur illusoire, mêle écriture et dessin, travaille la texture, ramène sur le même plan des éléments du passé (le sien et celui des États-Unis, de New York ou de Paris) et du présent. Il joue donc sur la relation, le dialogue, la communication-sujet qu'il reprend dans d'autres toiles de 1931, comme Radio Tubes, Rose Art Museum, Brandeis University, Waltham, Massachusetts), Television (collection privée), ou dans son projet History of Communications, 1939 (dont une étude était exposée à l'Orangerie dans le cadre de l'exposition The Age of Anxiety) -et la multiplicité des voix.

\section{Une esthétique de la communication : une abstraction paradoxale}

La fragmentation dont je parle plus haut est contredite par le travail sur la couleur, entamé dans des toiles précédentes, et poursuivi par la suite. En effet, les formes peintes sont reliées par l'emploi de rappels de couleur. La palette de Davis est ici réduite : il se contente de variations sur les couleurs primaires : les bleus allant au vert (avec le bleu-vert du faux cadre qui entoure la toile et en dédouble le cadre physique), les rouges allant vers les bruns (obtenus en mélangeant le rouge et le vert) et les orangés (rouge et jaune), et le noir et le blanc cassé du fond (ce qui revient pour l'essentiel à une palette autour des couleurs des drapeaux américain et français). Les couleurs éclatantes ne sont pas toujours pures : elles sont des mélanges, de couleurs primaires entre elles, ou de couleurs primaires éclaircies ou assombries par le rajout de blanc ou de noir. Si la couleur contribue à l'illusion de profondeur produite par l'emploi d'éléments verticaux (avec l'élément central de l'hôtel ou les trois troncs d'arbre), elle crée également une unité ainsi qu'une égalité entre les différents éléments de la composition, qui trouvent une affinité inattendue (à la manière des toiles surréalistes) de par leurs couleurs.

Déjà, dans les Egg Beaters de 1927-28, l'artiste cherchait à réduire l'objet, le batteur d'œufs, à une série de formes mises en tension par le biais du jeu des couleurs. Davis explique dans son autobiographie parue en 1945 que ce qui l'animait, c'était la recherche d'une dynamique des relations entre " espaces-couleur " sur la toile (" the dynamics of the actual color-space relations on the canvas », Davis, 1945, 24). Dans New York-Paris No. 3, les couleurs créent des espaces, des formes, qui entrent en collision, et cette interaction dynamique se fait d'une part entre les formes au sein de l'espace défini par la toile, mais également entre ces formes et le fond, l'arrière-plan, qui malgré 
sa couleur, n'est pas neutre: Davis l'utilise comme espace-couleur supplémentaire mettant en scène cette dynamique de la tension.

La couleur crée un fil conducteur dans une œuvre où d'autres fils conducteurs sont manifestes, comme le lien entre ce tableau et les précédents, et le cadre peint est alors un élément ironique qui indique une volonté de contenir le propos dans la toile, alors que tout ce qui y figure renvoie à d'autres œuvres, d'autres espaces peints, et d'autres réalités. Autre forme de contrepoint. En 1935, Davis écrit, au sujet du peintre régionaliste Thomas Hart Benton, qu'un art valable ne peut être créé que par des artistes qui envisageaient leur environnement non pas de façon isolée, mais en relation avec le tout («artists who perceive their environment, not in isolation, but in relation to the whole ", cité in Sims, 1991, 61).

17 Ici, le travail de Davis porte aussi sur le lien entre l'artiste et son environnement. D'une part, on l'a vu, les éléments de New York-Paris No.3 semblent isolés, mais ils sont en fait reliés entre eux et avec le reste de l'œuvre de Davis. Le tableau communique avec les autres toiles de la série, mais aussi avec des toiles d'autres époques. Elle communique aussi avec les mouvements artistiques européens (français) que sont le surréalisme ou le cubisme. Cette communication formelle s'ajoute à un dialogue avec l'époque. Autre forme de multiplicité des voix. En effet, le choix des éléments qui entrent dans cette composition n'est pas anodin. Les références au monde des transports routiers sont nombreuses (voir l'avion et le mât du voilier), mais Davis fait aussi allusion aux échanges de marchandises (le marché) ou de produits financiers (la bourse de New York), ou encore au transport du courrier ou de l'électricité (ou du télégraphe/ téléphone). L'hôtel est une étape pour le voyageur, le fauteuil et les chaises lui permettent de se reposer. Et ces éléments semblent renvoyer plutôt aux années folles qu'aux années de crise: l'essor de l'industrie automobile, de la société de consommation, les speakeasies sont autant de signes d'une Amérique florissante.

Mais le mouvement auquel Davis nous invite au début avec sa flèche (en suivant le sens conventionnel de la lecture) est mis en tension par le jeu entre la toile et son contexte. Les chaises retournées et les meubles empilés semblent indiquer qu'ils ne servent plus. Le garage est à la fois un obstacle (le bord du trottoir) et un frein; et le public américain savait que l'industrie automobile avait vu ses ventes chuter de plus d'un tiers dans les trois mois ayant suivi le krach de Wall Street. La dernière vignette est d'ailleurs celle de la bourse de New York, que le public américain aurait reconnue. La scène américaine que Davis veut s'approprier ici, pour ne pas la laisser aux seuls artistes régionalistes, est une scène de crise. Le mouvement est interrompu, le cadre empêche de circuler, et la bourse de New York, vue de profil, est inaccessible : on ne peut y entrer, et elle constitue le point final du trajet proposé par Davis. Finalement, le sens unique se termine en impasse. Cette toile est donc, aussi, sur l'anxiété face à la crise de $1929^{4}$.

Face à cette impasse, Davis se lance dans la série des New York-Paris, qui ramène dans un même espace (la toile, son titre, et celui de l'exposition) les deux villes et les deux expériences visuelles et esthétiques de l'artiste. La série reprend des éléments des toiles peintes à Paris, et donc elle renvoie à ce séjour de 1928-29 où Davis a été confronté à un nouvel environnement visuel, et où il a pu rencontrer des artistes dont il avait découvert le travail à New York dans les années précédentes, notamment Fernand Léger. Cette affinité entre ce que Davis recherche, un certain ordre, une objectivité qui dépasse la reproduction du réel, notamment à travers l'emploi des couleurs, et ce que les artistes européens faisaient alors est un premier pas, à travers une affiliation à la 
France, en direction d'un décentrage qui mène Davis vers une esthétique moderniste transnationale, dans un monde de l'art qui, déjà, devient mondial. Davis américanise cette démarche esthétique européenne, le modernisme lui apparaissant comme une esthétique du passage et de la connexion.

Pourtant, Davis écrit qu'à Paris, il ne voulait pas peindre autre chose que "la réalité " de la capitale («Over there, the actuality was so interesting I found a desire to paint it just as it was », cité un Sweeney, 1945, 20). Mais cette réalité (ou cette factualité) dépasse le simple paysage pour intégrer l'actualité des recherches picturales contemporaines, notamment à Paris: pas de fuyantes pour créer l'illusion de profondeur ou de "réalisme ", mais un travail sur la profondeur créée par la couleur (avec les arbres, ou l'hôtel, ou encore l'automobile entre les deux pompes à essence) et le cadre peint en bordure de l'œuvre, ici comme dans les toiles parisiennes, qui souligne la planéité de la toile et donc l'illusion de ce qui y figure/ y est figuré.

21 Ce travail contradictoire, cette confrontation et cette mise en tension entre la représentation traditionnelle et les questions esthétiques plus contemporaines est commun à toutes les toiles parisiennes de Davis, et à la série des New York- Paris. Ce qui semble compter, c'est la construction (à travers les formes perçues par l'artiste, y compris les formes créées par les lettres des mots qu'il intègre dans cette composition) et la destruction simultanée de l'illusion, qui mène à l'abstraction.

En fait, Davis part d'un constat : la coexistence dans le paysage urbain parisien du passé et du présent («There was so much of the past, and the immediate present, brought together on one plane ", Sweeney, 1945, 19). Paris est une zone de contacts, une zone de simultanéité. Et les toiles qui y sont produites ou qui s'en inspirent sont elles-mêmes des zones de contact et de simultanéité. Mais elles sont également, et paradoxalement, des zones de réticence, où l'insistance simultanée sur des éléments disparates, ou sur l'illusion de profondeur et la surface plane, crée un espace incertain. Ce qui semble intéresser le peintre, c'est la dualité (des façades du marché et des gratte-ciels ou de l'hôtel, de la surface et de la profondeur, de l'arrière-plan et du premier plan, de l'écriture et de la peinture, et de la peinture et du dessin), la juxtaposition de plans et de plans-couleur (ce qu'il appelle « color-shape ») ou d'éléments parisiens et américains qui flottent sur un fond neutre.

Mais à travers cette dualité, la toile (comme les toiles parisiennes qui la précèdent) est un lieu de passage et de rencontre : entre le paysage et l'expérimentation formelle, les esthétiques européennes (le cubisme, Duchamp dans New York-Paris No.1, Léger) et le travail d'un Américain à Paris. Il s'agit aussi du passage d'un même élément, narratif ou structurel, d'une toile à une autre. On l'a vu, les toiles de Davis communiquent entre elles et cette inter-iconicité interne est transformatrice: elle introduit le mouvement dans le travail du peintre, et crée des constellations d'œuvres reliées en un système de communication. Si les juxtapositions dans New York-Paris No.3 font se rencontrer et communiquer le passé, le présent (et même l'avenir, à travers la reprise d'éléments dans des toiles qui suivent), le travail d'abstraction de Davis tend vers une déterritorialisation et une atemporalité qui permettent à l'artiste de réaliser une œuvre paradoxalement universelle à partir d'éléments vernaculaires. En 1935, il déclare par exemple :

My paintings are never paintings of Paris, Gloucester, or New York. They are coherent formal symbols with a meaning of Emotional Equilibrium which is independent of Time or Place. (Davis, SDP 15 décembre 1935). 
En 1943, il reprend la même idée. La peinture, pour être objective et universelle, audelà du temps et de l'espace, et provoquer une réaction intellectuelle, doit se dissocier de l'expérience commune, du subjectif :

Today, I disassociate the painting experience from general experience and attain a universal and objective statement that transcends the subjectively particular. The emotion is given an intellectual currency beyond time and place (Davis, SDP, juillet 1943).

Les toiles de la série New York-Paris sont donc, bien que situées dans l'espace géographique et dans le temps de la Grande Crise, une étape vers une œuvre dont l'ambition est d'être universelle. Cet universalisme passe par une esthétique cosmopolite.

\section{Dialogue transatlantique : vers une esthétique cosmopolite} médiation entre préoccupations formelles (relations spatiales, relations entre couleurs) et figuration, faisant le pont entre perception et conceptualisation (c'est d'ailleurs comme cela qu'il définit le cubisme: "Cubism is the bridge between percept and concept ", Davis, SDP 1918). Après les trois toiles de la série New York-Paris, de plus en plus, il reprend des motifs de toiles anciennes et les retravaille pour créer une nouvelle oeuvre, en renouvelant les couleurs, en simplifiant ou en supprimant les lignes, en changeant les titres, en brouillant les pistes qui ancreraient son travail dans le réel. Il explique ainsi en septembre 1940: «Abstract painting recombines (...) selections of color and shape into a new unity, which never existed in nature but is a new part of nature » (cité in Kelder, 1991, 28). Et ailleurs : " The new is a recovery not a discovery " (Davis, SDP, 30 juin 1956), la nouveauté vient non pas de la découverte, mais d'une sorte de redécouverte.

L'une des stratégies de Davis, dans cette démarche de re-travail et de réévaluation, est de «franciser » ses œuvres. En effet, après la série des New-York-Paris, Davis introduit dans ses œuvres, vers le début des années 1950, des mots français, ou des titres en français (bien qu'il ait toujours affirmé ne pas parler la langue) alors qu'elles partent de paysages américains. Par exemple, Semé de 1953 (Metropolitan Museum of Art, New York) reprend une nouvelle fois le paysage de Gloucester, peint en 1922. La première œuvre est une sorte de pseudo-collage, où Davis explore les relations entre les couleurs et la création de profondeur à travers d'une part la juxtaposition de formes de couleurs différentes, et d'autre part un traitement différentiel de la matière (transparence/ épaisseur). Il explique ce processus en utilisant le terme espace-couleur, ou «colorspace $»^{5}$. Chaque fois que Davis revient sur un tableau, il crée un espace relationnel résultant de la translation d'une toile vers l'autre d'un noyau schématique ou thématique, créant ainsi des ensembles ou constellations d'œuvres. Le titre français apporte des éléments théoriques au tableau: Davis sème, dissémine, superpose des formes de couleurs de façon qui semble aléatoire à partir d'une composition préexistante, et par là, reproblématise la composition.

Les titres numérotés des toiles New York-Paris, anodins mais évocateurs d'une série, créent un lien entre l'œuvre de ce peintre américain et une autre nation. Ainsi, pour reprendre l'idée de Rebecca Walkowitz (2006), Davis sape, désamorce le discours 
régionaliste nationaliste autour de l'art. L'association de la capitale française et de la ville cosmopolite de New York, d'éléments des deux pays, mais aussi de paysages et de mots américains dé-hiérarchise le champ artistique national, contestant son caractère normatif, et le système de pouvoir qui en découle, créé par certains artistes et critiques d'art aux États-Unis. La présence de mots ou d'éléments français (pour le titre d'une œuvre, ou dans son contenu) place ces tableaux dans une stratégie de la confrontation où Davis s'oppose aux nouvelles esthétiques dominantes qu'il renie.

Les toiles de Davis, et notamment New York-Paris No.3, sont donc un lieu d'échange entre les formes inventées par l'artiste à partir de paysages et mots américains et parisiens. Davis y montre ses allégeances multiples à une esthétique européenne (le cubisme), et à un environnement visuel à la fois américain et français. Travaillant dans la dualité, il parvient, à mon sens, à un cosmopolitisme moins universel que critique : cette série, comme ses autres toiles sont certes internationales (l'une d'elles, de 1958-60 porte le titre International Surface No.1), et Davis refuse les normes esthétiques imposées par le champ artistique, dans les années 30, puis encore plus pendant la Guerre Froide. Ce travail aboutit à ce qui a été appelé un cubisme vernaculaire. L'œuvre de Davis est aussi le lieu d'explorations formelles sur la perception et la communication. Mais surtout, cette utilisation de données locales et françaises est critique, à l'égard du champ artistique américain comme d'un système social et économique en faillite. De plus, chaque œuvre, en reprenant des motifs d'autres toiles, est un examen critique où Davis innove et renouvelle son propre travail. Cette ré-évaluation personnelle débute notamment avec New York-Paris No.3. Son cosmopolitisme, donc, s'affirme dans la confrontation de sa mémoire et de son environnement direct, du passé et du présent, de la France et de l'Amérique, dans une démarche esthétique de la mise en rapport (confrontation, connexion, mélange, communication... translation) critique.

\section{Conclusion}

New York-Paris No.3 est à la fois une prise de position esthétique, un commentaire sur l'impasse où se trouvait le pays, et un commentaire critique face au monopole qu'entendaient avoir en ce début de décennie les peintres régionalistes sur la représentation de la scène américaine. Le tableau est un résumé des préoccupations esthétiques et idéologiques de Davis, et place l'artiste dans un dialogue transatlantique: d'une part, sur le plan esthétique, il prend à son compte les questionnements des cubistes et des surréalistes et les réexamine. Il s'oppose ainsi à l'esthétique communiste, défendue par la gauche aux États-Unis, et qui rejetait de plus en plus les esthétiques modernistes, et notamment l'influence parisienne ${ }^{6} \mathrm{Cela}$ lui permet, d'autre part, de s'opposer au nationalisme d'autres mouvements artistiques, à travers une représentation critique du paysage urbain américain.

Ce nationalisme résulte de la politique de propagande du président Woodrow Wilson après l'entrée en guerre des États-Unis en 1917, et se traduit par la «terreur rouge » et le nativisme des années 1920. Il se retrouve dans l'évolution des positions du critique d'art Thomas Craven sur les esthétiques européennes, qui partent d'une adhésion au modernisme en 1913 à la défense d'un art autochtone libéré du joug européen au début des années 1930 : «Art is the child of new understandings and fresh appreciations of common things, and it is beyond the reach of those who are slaves to the impulses of others » (Craven, 1929, 63). Davis répond dans cette toile en partant d'objets banals et 
montre l'échec du système financier d'un pays dont la politique, depuis le début des années 1920, est fondée sur le laissez-faire, tout en gardant un attachement aux questions formelles posées par les modernistes parisiens. Il montre ainsi qu'une esthétique née outre-Atlantique peut s'adapter au contexte américain, et qu'il est possible de dépasser la « contradiction entre formalisme et engagement social » dans le contexte de la Grande Dépression, puis tout au long de sa carrière (De Chassey, 2001, 25). De manière générale, pour Davis, la liberté d'expression ou de choix esthétique, et donc la possibilité de se référer au langage plastique parisien, sont une réponse aux nationalismes à différentes époques et dans différents contextes. Ainsi, deux décennies plus tard, en plein McCarthysme, écrivait-il en 1953 à sa galeriste Edith Halpert :

I am strictly a European (French, that is) man myself, altho forced by birth and circumstance to live in the American Art Desert as exile. And then of course the 'Europe' I mentally dwell in no longer exists in actuality (cité in Lane, 1991, 79).

\section{BIBLIOGRAPHIE}

ARNASON, H.H., Stuart Davis, catalogue d'exposition, Minneapolis, Walker Art Center, 1957.

CRAVEN, Thomas, « The Curse of French Culture », Forum, 82, juillet 1929, 63.

DAVIS, Stuart, « Autobiography », American Artists Group Monograph, 6, 1945, n.p., reproduit in Diane Kelder, ed., Stuart Davis, New York, Washington et Londres : Praeger, 1971, 23-24.

--- Stuart Davis Papers, Harvard Art Museums, Fogg Art Museum, Cambridge, Mass.

De CHASSEY, Eric, « Pourquoi n'y a-t-il pas eu de grand art américain ? », in Made in America, 1908-1947, Paris, RNM, 2001, 21-29.

DELANTY, Gerard, « The Cosmopolitan Imagination : Critical Cosmopolitanism and Social Theory ", British Journal of Sociology, vol. 57, n 1, 2006, 25-47.

HAGELSTEIN MARQUARDT, Virginia, « 'New Masses' and John Reed Club Artists, 1926-1936 : Evolution of Ideology, Subject Matter, and Style », The Journal of Decorative and Propaganda Arts, Vol. 12 (Spring 1989), 56-75.

KELDER, Diane, « Stuart Davis and Modernism, an Overview », in Lowery Stokes Sims, ed. Stuart Davis, American Painter, catalogue d'exposition, New York, Metropolitan Museum of Art, 1991, 17-30.

LANE, John R., « Stuart Davis in the 1940s », in Lowery Stokes Sims, ed., Stuart Davis, American Painter, catalogue d'exposition, New York, Metropolitan Museum of Art, 1991, 70-81.

SIMS, Lowery Stokes, ed., Stuart Davis, American Painter, catalogue d'exposition, New York, Metropolitan Museum of Art, 1991.

SWEENEY, James Johnson, Stuart Davis, catalogue d'exposition, New York : MoMA, 1945.

WALKOWITZ, Rebecca L., Cosmopolitan Style : Modernism beyond the Nation, New York : Columbia University Press, 2006. 
WHITNEY MUSEUM OF AMERICAN ART, Stuart Davis : an American in Paris, catalogue d'exposition, New York : Whitney Museum of American Art at Philip Morris, 1987.

\section{Fuvres citées}

1917 : Garage N.2, huile sur toile (h/t), collection privée.

1922 : Landscape, Gloucester, (h/t), collection privée.

1925 : Early American Landscape, (h/t), Whitney Museum of Art, New York.

1927-1928 : Egg Beater N.1, (h/t), Whitney Museum of Art.

Egg Beater N.2, (h/t), Amon Carter Museum of American Art, Fort Worth, Texas;

Egg Beater N.3, (h/t), Museum of Fine Arts, Boston, Mass.;

Egg Beater N.4, (h/t), The Phillips Collection, Washington D.C.

1931 : New York-Paris N.3, (h/t), Estate of Stuart Davis.

Still Life, Radio Tubes, (h/t), Rose Art Museum, Brandeis University, Waltham, Mass.

Television, (gouache sur papier), collection privée.

1953 : Semé, (h/t), Metropolitan Museum of Art, NY. LIEN www.metmuseum.org/art/collection/ search/488756

1958-60 : International Surface, N.1, (h/t), National Museum of American Art, Smithsonian Institution, Washington D.C. LIEN: https://americanart.si.edu/artwork/intl-surface-no-1-6353

\section{NOTES}

1. Cette double identité remonte à l'époque du séjour du peintre à Paris : il exposa des œuvres parisiennes à la Downton Gallery de New York, d'abord dans le cadre de l'exposition de janvier 1929, «American Painters in Paris », puis à l'automne de la même année dans l'exposition «Hotels and Cafés » (Whitney).

2. Par exemple, à l'occasion d'une exposition des œuvres des membres du Group of American Painters in Paris, le critique d'art Helen Appleton-Read écrivait dans le populaire Art Digest du 1er mai 1927 qu'ils produisait un art «hybride», l'expatriation ayant sur leur travail un « effet stérilisant »(p.14).

3. New York-Paris No 1, 1931, se trouve au Musée d'Art de l'Université d'Iowa, et New York-Paris No 2, 1931, au Portland Museum of Art (Maine).

4. Davis avait exposé au sein des John Reed Clubs de New York, fondés par d'anciens éditeurs de The Masses et ayant pour but de promouvoir l'art dans les quartiers pauvres. En 1931, le Club présenta à l'exposition annuelle de la Society of Independent Artists une toile intitulée Washington Market, œuvre collective représentant le président Herbert Hoover conduisant une charrette de poisson portant le mot «USA ». Je pense que Davis connaissait cette œuvre, et que le marché, ici, est un clin d'oeil à l'art de propagande des artistes membres du John Reed Club. (Voir Hagelstein Marquardt, 1989)

5. Il explique en 1957 : "Every time you use a color you create a space relationship.... I simply called the things that happen when you use two colors, and the process of drawing and painting, a color-space event » (Arnasson, 1957, 44)

6. Lors de la conférence de Kharkov de 1930, il fut décidé que le seul art acceptable était l'art révolutionnaire qui devait faire référence à la lutte des classes dans un langage pictural lisible. 


\section{AUTEUR}

\section{KAMILA BENAYADA}

Université d'Orléans, PoLeN 